Ophthalmologica 1969;157:401-406

\title{
Marc Amsler
}

\section{1-1968}

Le 6 mai 1968 Marc Amsler, un des éditeurs de ce journal, a été enlevé par une défalliance cardiaque à $\Gamma$ âge de 77 ans.

Les lecteurs d'Ophthalmologica se souviennent de ce Maître qui a si souvent écrit pour eux. Sa plume alerte, sa langue concise, ses idées claires donnaient à ses articles et à ses communications une tournure particulière. II arrivait en quelques pages elegantes à dé-

402

Marc Amsler, 1891-1968

velopper un sujet, ne donnant que Tessentiel. II laissait à d'autres le soin de se débattre avec les problèmes marginaux.

Ces dons de clartź et d'organisation lui ont permis de former au cou $\gamma \mathrm{s}$ des 25 ans de sa carrière de professeur, de très nombreuxi étudiants et élèves qui sont restés fidèles à $\Gamma$ ophtalmologie. Et ceux de ses élèves qui continuent à pratiquer se rappellent avec reconnaissance des leçons qu'il leur a données. D'abord $\Gamma$ examen devait être complet et les notes cliniques tenues régulièrement à jour. II fallait dessiner ou photographier ce que $\Gamma$ on voyait, mesurer ce qui était mesurable et mener une thérapeutique active aussi bien sur le plan médicamenteux que psychologique.

Longtemps il a con-trôlé deux à trois fois par semaine le travail des assistants, exami-nant luimême tous les malades de la clinique.

C'est que d'abord il avait une vocation de médecin, d'oculiste. La recherche était certes importante pour lui mais elle devait être subordonnée à la clinique. C'est dans cet esprit qu'il avait fait ins-crire dans ses salles d'opération les deux adages suivants : « Pri-mum humanitas » et « Salus aegroti suprema lex ».

Chirurgien de grande classe, il ne laissait rien au hasard: Гin-dication opératoire, les instruments et tous les details de la salle et de Гéquipe opératoire étaient minutieusement mis au point.

C'était un plaisir et une excellente leçon de le voir opérer : on voyait se dérouler des images aussi claires que celles d'un atlas : elegance, precision, geste decide. Lors de complications, il ne perdait jamais son calme. Sa grande experience lui permettait le plus souvent de se sortir des situations les plus délicates. C'était un maître patient qui a passé un temps incalculable à assister ses élèves. II a forme de bons opérateurs, conscients de leur responsabilité.

Marc Amsler a commence sa carrière universitaire à Lausanne. Tout d'abord élève du bactériologue Galli-Valerio, du chirurgien Cesar Roux, puis des ophtalmologues Haab à Zurich et Eperon à $\Gamma$ Hôpital Cantonal de Lausanne, il est devenu par la suite élève de Jules Gonin et médecin adjoint de ГHôpital ophtalmique. En 1928 il a été nommé privat-docent à $\Gamma$ Université de Lausanne.

A ce moment, seul un petit cercle de jeunes oculistes admettait la justesse des idées de Gonin sur le traitement du décollement de rétine. Parmi les élèves de Gonin, principalement aux côtés d'Ar-ruga de Barcelone et de Weve d'Utrecht, Amsler a joué un role important pour la diffusion des idées et de la technique opératoire de son maître. C'est son talent d'organisation, de classification et 
Marc Amsler, 1891-1968

403

enfin de pedagogue qui a permis à de nombreux confreres de se familiariser avec les idées de Gonin et leur a donné le courage de tenter à leur tour cette intervention delicate.

En 1935 Marc Amsler a succédé à son Maître à la chaire d'oph-talmologie de $\Gamma$ Université de Lausanne et peu après à la direction de ГHôpital ophtalmique. Au cours des 9 ans qu'il y a passes, il a fallu entreprendre d'importantes reparations que la deuxième guerre mondiale ont fortement ralenties.

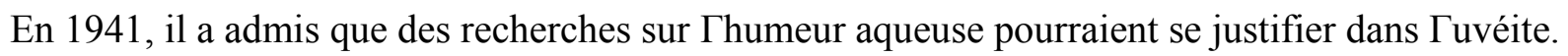
II avait au debut de nos discussions pensé que la ponction de la chambre antérieure était trop dangereuse, puis a change d'avis et a fait fabriquer par Grieshaber $\Gamma$ excellente lance-canule qui porte son nom. Des milliers de ponc-tions au cours de 20 ans lui ont permis ainsi qu'à ses collaborateurs de faire de fructueuses recherches sur $\Gamma$ humeur aqueuse patholo-gique. Elles sont à Torigine des 94 travaux dont une monographic iniportante et un rapport à la Société Française d'Ophtalinologie qui sont sortis de ses cliniques de Lausanne et de Zurich. C'est aussi à Lausanne qu'ont commence sur Tinstigation de Hessberg les recherches sur le passage de la fluorescéine du sang dans Thumeur aqueuse.

Peu après avoir été nommé professeur ordinaire à Lausanne, en 1943, Marc Amsler a accepté Гappel de $\Gamma$ Université de Zurich. II a en quelques mois opéré une metamorphose étonnante, passant du français à $\Gamma$ allemand pour ses cours. En outre il avait laissé à Lausanne une clientele très iniportante et accaparante. Le passage à Zurich lui a permis, au cours des premieres années, de se débarras-ser de cette charge et d'avoir beaucoup plus de temps pour la preparation et la discussion des travaux en cours sur le décollement de rétine, la ponction de la chambre antérieure et la barrière hémato-oculaire.

A côté de ses travaux cliniques, il s'est occupé des recherches qui Гinléressaient, faisant installer entre autres un laboratoire photographique niodèle et luttant pour obtenir les modestes credits nécessaires aux recherches de ses assistants. A part des incursions sporadiques dans d'autres domaines, Amsler ne s'est intéressé qu'à quelques themes de recherche tout au long de sa carrière : le décollement de rétine, le kératocône, Гuvéite et son diagnostic ćtio-logique (humeur aqueuse et barrière hémato-oculaire), les affections de la macula et leur traitement.

404

Marc Amsler, 1891-1968

Nous avons déjà parlé de Гuvéite et du décollement de rétine. Arrêtons-nous un instant au kératocône. C'est en 1930 qu'il a publié sa premiere communication sur la kératophotographie et Tappareil qu'il avait fait construire par Zeiss et qui lui permettait de relever avec precision les courbes de niveau de la cornée ainsi que les axes vertical et horizontal. Grace à cet appareil il a obtenu des données objectives qui lui ont permis de suivre photogra-phiquement revolution du kératocône, de déceler ces manifestations à Гétat fruste (1937) et de prouver la presence de cas frustes dans les families où se rencontrait un cas avancé (1938). II a patiemment collationné les cas et en a réuni plus de 700 dont beau-coup ont été suivis pendant des décennies. II a publié une douzaine de travaux et a fait faire des theses sur ce sujet. La monographic qu'il se proposait d'écrire n'a pas vu le jour, mais ce sujet con-tinuait à le préoccuper. En février 1968, malade, il m'avait écrit: « Le kératocône m'apparait comme un petit point lumineux au bout d'un long tunnel.» Au matin de sa mort il a dit à sa femme : «J'ai terminé cette nuit mon article (sur le kératocône) en anglais, il ne me reste plus qu'à Гécrire.» II faudra rechercher dans ses notes et dans cette incomparable collection ce qu'il voulait encore nous reveler. 
Amsler s'est aussi penché pendant des années sur les problèmes poses par Гexamen objectif et subjectif des lesions de la macula, par leur étiologie et par leur traitement. II nous a donné la grille qui porte son nom et permet d'étudier les métamorphopsies, les scotomes relatifs ou absolus de la region maculaire, ainsi que leur evolution fonctionnelle. L'idée de cette grille lui est venue d'un carré quadrille de $10^{\circ}$ de côté place au centre du périmètre de Landolt. Ce dernier n'a jamais publié le travail explicatif qu'il pro-mettait pour plus tard. Amsler a fait une etude de longue haleine sur Гutilité de ce carré pour Гétude des lesions maculaires et para-maculaires. II a publié plusieurs travaux, son fils Etienne a fait une these remarquée sur ce sujet et le dernier article de sa carrière, paru dans la Revue Chibret, y est consacré ; c'est done à juste titre que le quadrillage qu'il nommait le carré de Landolt est devenu par la suite la grille d'Amsler. On pourrait s'étonner du nombre relativement modeste des travaux (un peu plus de 120) qu'un homme aussi actif a signés au cours de sa carrière. II faut dire à ce propos que, bien qu'il se soit intéressé, bien qu'il ait inspire, qu'il ait lu et qu'il ait propose

Marc Amsler, 1891-1968

405

des modifications à la plupart des articles ou communications sortant de sa clinique, il ne les a pas signés pour autant avec leur auteur, sauf dans certains cas. De 1945 à 1961 par exemple, il a signé 59 articles seul, 26 seulement avec des collaborateurs tandis que ses assistants publiaient en outre 151 travaux signés de leur seul nom et qu'il sortait 40 theses de la clinique de Zurich. Encore faut-il remarquer qu'Amsler n'a pas conserve tous les articles publiés dans la presse non spécialisée ou ne traitant pas de sujets ophtalmologiques. Le contenu lui tenait plus à cæur que le nombre et il reste là un travail bibliographique à accomplir.

Ses travaux et ses talents de conférencier ont valu à Marc Amsler des titres honorifiques. Lauréat de $\Gamma$ Université de Lausanne en 1917, il a reçu la médaille Bowman à Londres en 1948 pour la Bowman-Lecture (New clinical aspects of the vegetative eye). II a été nommé membre d'honneur des sociétés d'ophtalmologie de Belgique, du Boyaume-Uni, de Cordoba (Argentine), de Grèce et de Suisse. II était aussi membre correspondant de la Société d'Ophtalmologie de Paris.

Parler des qualites médicales, professorates et scientifiques de Marc Amsler est insuffisant. Ses qualites humaines étaient au premier plan. Son charme et la peine qu'il se donnait pour tous ceux qui lui demandaient conseil lui ont valu beaucoup d'amis : malades, confreres, artistes. Sous un aspect conciliant et doux, il cachait une volonté de fer. Sans vouloir heurter de front les recalcitrants, il savait par diplomatie et resistance « élastique » (pour employer un terme militaire) les faire passer par où il le voulait. Un esprit d'organisation remarquable lui a permis d'abattre un travail énorme malgré le temps mesuré dont il disposait; très consciencieux, il a souvent travaillé jusqu'à la limite de ses forces. C'est pourquoi il exigeait aussi beaucoup de ses collaborateurs. Issu d'une famille très pieuse, il a lui-même toujours été croyant. C'était un laïc engage au service de $\Gamma$ église et il a rendu de grands services à ses paroisses successives : tenant Torgue fidèlement le dimanche à Lausanne, et, tout récemment, choisissant un orgue pour la chapelle protestante de Sierre, où il s'était retire sans rester pour autant inactif. II y a pendant quelques années dirigé les premiers travaux de la réédition du " Lehrbuch der Augenheil-kunde » jusqu'à ce que la maladie le force à s'arrêter. Marc Amsler aimait aussi les arts, la musique surtout et la peinture. II était aussi collectionneur et il avait, au cours des années, rassemblé des 406 Marc Amsler, 1891-1968 autographes intéressants d'hommes scientifiques, d'artistes et même de quelques homines politiques. 
Mais plus que tout, c'était sa famille qui lui tenait à cæur. II a consacré à sa femme et à ses enfants tout le temps qu'il ne devait pas à son travail. C'est auprès d'eux qu'il allait reprendre des forces.

S. Ses malades, ses amis, ses élèves ne sont pas près d'oublier cemédecin, cet ami, ce maître qui d'une façon ou d'une autre amarque leur vie d'une manière durable.

Florian

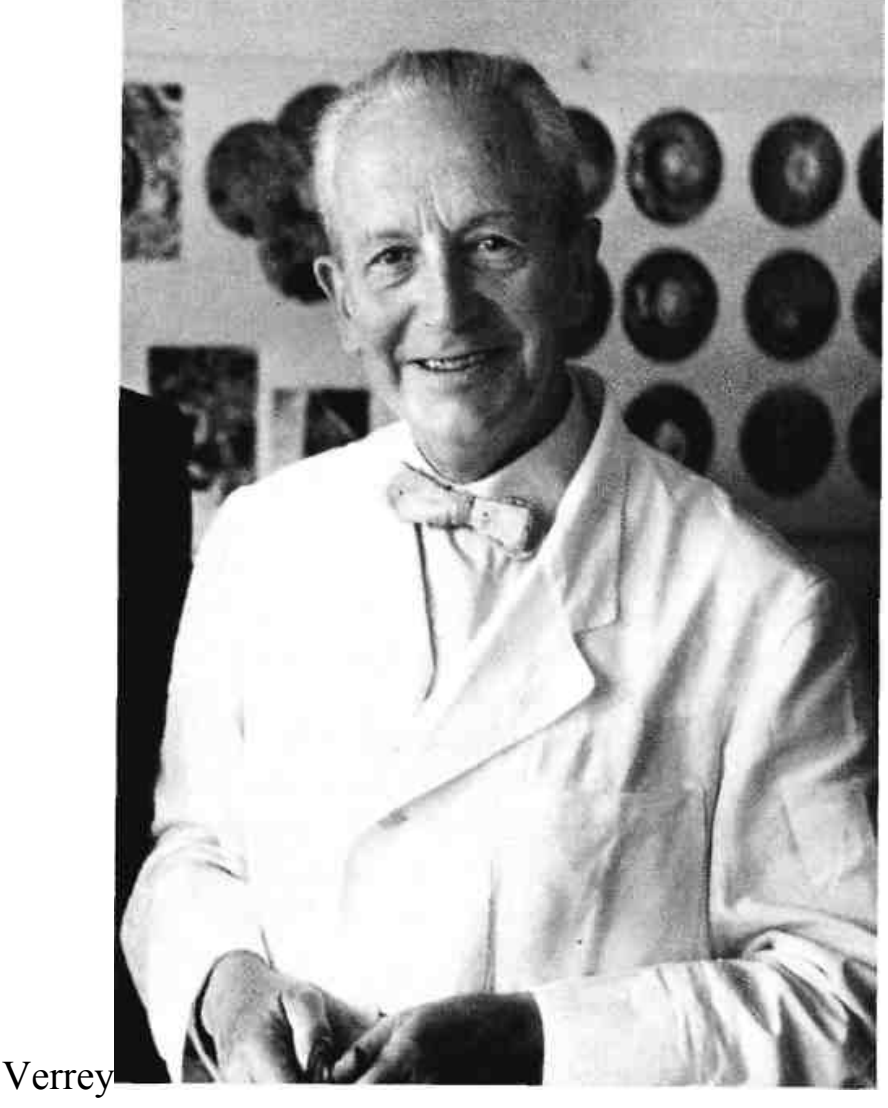

